\title{
Insight into the structure of black coatings of ancient Egyptian mummies by advanced EPR spectroscopy of vanadyl complexes.
}

Charles-Emmanuel Dutoit

Chimie-ParisTech, PSL University, CNRS, Institut de Recherche de Chimie-Paris https://orcid.org/00000002-2181-4113

\section{Laurent Binet}

Chimie-ParisTech

Hervé Vezin

Laboratoire de Spectrochimie Infrarouge et Raman

Océane Anduze

Chimie-ParisTech

\section{Agnès Lattuati-Derieux}

Centre de Recherche et de Restauration des Musées de France

Didier Gourier ( $\nabla$ didier.gourier@chimieparistech.psl.eu )

didier.gourier@chimieparistech.psl.eu

\section{Article}

Keywords: EPR spectroscopy, mummies, ENDOR, pulsed EPR

Posted Date: October 21st, 2021

DOl: https://doi.org/10.21203/rs.3.rs-960462/v1

License: (9) This work is licensed under a Creative Commons Attribution 4.0 International License.

Read Full License 


\title{
Insight into the structure of black coatings of ancient Egyptian mummies by advanced Electron magnetic resonance of vanadyl complexes.
}

\author{
Charles E. Dutoit, ${ }^{\dagger}$ Laurent Binet, ${ }^{\dagger}$ Hervé Vezin, ${ }^{\&}$ Océane Anduze, ${ }^{\dagger}$ Agnès Lattuati-Derieux, ${ }^{\ddagger}$ \\ Didier Gourier ${ }^{*}$ \\ † Chimie-ParisTech, PSL University, CNRS, Institut de Recherche de Chimie-Paris (IRCP), F-75005 Paris, \\ France \\ ‡ Centre de Recherche et de Restauration des Musées de France (C2RMF), Palais du Louvre, F-75001 Paris, \\ France \\ \& Université de Lille, CNRS, UMR8516-LASIRE, F-59000 Lille, France.
}

\begin{abstract}
:
Advanced EPR techniques such as ENDOR and pulsed EPR are used to investigate the enigmatic black coatings of ancient Egyptian mummies, consisting in a complex and heterogeneous mixtures of conifer resins, wax, fat and oil with variable amounts of bitumen. Natural bitumen always contains traces of vanadyl porphyrin complexes that we used here as internal probes to explore the nanoscale environment of $\mathrm{V}^{4+}$ ions in these black coatings by hyperfine spectroscopy. Four types of vanadyl porphyrins were identified from the analysis of ${ }^{14} \mathrm{~N}$ hyperfine interactions. Three types (referred to as VO-P1, VO-P2 and VO-P3) are present in natural bitumen from the Dead Sea, among which VO-P1 and VO-P2 are also present in black coatings of mummies. The absence of VO-P3 in mummies, which is replaced by another complex VO-P4, may be due to its transformation during preparation of the black matter for embalming. Analysis of ${ }^{1} \mathrm{H}$ hyperfine interaction shows that bitumen and other natural substances are intimately mixed in these black coatings, with bitumen aggregate sizes not larger than a few nanometres.
\end{abstract}


Mummies and wooden coffins, funerary artifacts and panel paintings in ancient Egypt were often covered with enigmatic organic black materials, made of a heterogeneous mixture of natural substances such as fat, oil, wax, conifer or mastic tree resin, pitch, animal glue, plant gum and bitumen) in variable proportions. ${ }^{1-4}$ Those are characterized by a variety of molecular biomarkers identified mainly by gas chromatography - mass spectrometry (GC-MS). However, the presence or not of bitumen in these black materials has been the subject of a long controversy due to the fact that the analytical protocols used were often not well-adapted to the detection of bitumen, so that two opposite opinions have emerged among researchers analyzing these black coatings: those who did not believe in the presence of bitumen, ${ }^{5-7}$ and those who claimed its presence. ${ }^{1,8-12}$ With the detection of specific biomarkers (hopanes, steranes) and radiocarbon analyses (bitumen has lost its ${ }^{14} \mathrm{C}$ ), a consensus has recently emerged on the increasing presence of bitumen in embalming materials from the New Kingdom (ca 1550-1070 BC) to the Ptolemaï/Roman period ending in the 4th century AD. ${ }^{3}$ Despite the inestimable contribution of GC-MS for revealing the composition of these black coatings, this microdestructive technique requires preliminary steps of fractionation and separation, which exclude any direct, non-destructive identification of bitumen, so that structural information on this black material cannot be obtained at the nanometer scale. Such kind of information can only be obtained with non-destructive analysis techniques, i.e. which leave the samples intact. This is the case with magnetic resonance techniques because the low frequency electromagnetic fields (radiofrequency for NMR and microwave frequency for EPR) penetrate the whole sample and deposit a totally negligible energy in the material compared to the other spectroscopic techniques. Multinuclear magnetic resonance $\left({ }^{1} \mathrm{H}-\right.$ and $\left.{ }^{23} \mathrm{Na}-\mathrm{NMR}\right)$ of mummified tissues is mainly used in the imaging mode ${ }^{13,14}$ rather than the spectroscopic mode, ${ }^{15}$ owing to the rather low sensitivity and spectral resolution of NMR for these highly disordered solid materials. Electron paramagnetic resonance (EPR) is the electronic equivalent of NMR, and applies in the presence of unpaired electron spin density, i.e with electron spin $\mathrm{S} \geq 1 / 2$. The spectroscopic resolution of EPR is optimal when the paramagnetic entities (transition metal ions, radicals, ...) are magnetically diluted, which correspond to defects and impurities in material. ${ }^{16}$ It is well known that oil and bitumen contain organic radicals and porphyrinic complexes of vanadyl $\mathrm{VO}^{2+}$ ions ( $\mathrm{V}^{4+}$ ion, $3 \mathrm{~d}^{1}$ configuration). These very stable paramagnetic complexes are present mainly in asphaltene - the most refractory fraction of oil and bitumen - and can be considered as molecular markers of bitumen which can be detected with high sensitivity by EPR spectroscopy. ${ }^{17-20}$ Generally speaking, vanadyl porphyrins complexes (hereafter referred to as 
VO-P) are specific of oils and bitumen of marine origin, ${ }^{21-23}$ while carbonaceous radicals (hereafter referred to as $\mathrm{C}^{0}$ ) are present in all fossilized organic matters, whether of marine or terrestrial origin, ${ }^{24-26}$ and even in the extraterrestrial organic matters of the most primitive carbonaceous meteorites. ${ }^{27}$ Recently, we showed that EPR analysis of VO-P complexes and $\mathrm{C}^{0}$ radicals is a simple and nondestructive way (no sample preparation) to reveal the presence of bitumen in black coatings of Egyptian mummies, even in small amount. ${ }^{28}$ In addition to VO-P, the latter contain also non-porphyrinic $\mathrm{VO}^{2+}$ complexes (hereafter referred to as VO-nP), with four oxygen ligands in nearly square planar configuration. ${ }^{28}$ These VO-nP complexes are absent in Dead Sea bitumen used by Egyptians, and present in black coatings containing natural substances in addition to bitumen. We hypothesized that these VO-nP could result from the demetallation of VO-P of bitumen followed by the complexation of $\mathrm{VO}^{2+}$ by oxygenated functions of other components of the black matters.

However the resolution of EPR spectra of VO-P in such disordered materials is limited by the fact that the weak hyperfine (hf) interactions with other nuclei, namely ${ }^{1} \mathrm{H}(\mathrm{I}=1 / 2,100 \%$ abundance $),{ }^{14} \mathrm{~N}(\mathrm{I}=1,99.6 \%$ abundance $)$ and ${ }^{13} \mathrm{C}(\mathrm{I}=1 / 2,1.1 \%$ natural abundance $)$ are unresolved. These hindered hf interactions contain precious information about the structure of VO-P complexes, their environment and possibly on the degree of alteration of the black matters. This information can be recovered by the indirect detection of NMR transitions of magnetic nuclei in the environment of the unpaired electron spin. By this means, $\mathrm{VO}^{2+}$ of bitumen can be considered as internal probes which "see" their nuclear spin environment in a non-destructive manner.

Here we used Electron Nuclear Double Resonance spectroscopy in continuous-wave mode (cwENDOR) for ${ }^{1} \mathrm{H}$ nuclei, and HYperfine Sublevel CORrelation (HYSCORE) spectroscopy for ${ }^{14} \mathrm{~N}$ nuclei to study the same corpus of Egyptian black coatings than in the preliminary cw-EPR study. ${ }^{28}$ We found that proton ENDOR is sensitive to the bitumen content of the black matters and to the size of bitumen aggregates in the mixture, while HYSCORE of nitrogen reveals the presence of different types of VO-P complexes in the material. Special attention was given to the black coating of a human mummy of unknown origin (Hum 3), but whose EPR characteristics differ clearly from coatings of the other studied mummies. ${ }^{28}$

\section{Results and discussion}

The origins of black coatings are summarized in Table 1, and samples studied by EPR are described in more details in Table S1 and Fig. S1. Three samples were taken from the coating 
of an anthropomorphic coffin (Hum 1) dated from the Ptolemaïc period, and two human mummies (Hum 2 and Hum 3) dated from the third Intermediate period. Four samples were taken from animal mummies dated from the same periods as human mummies, three rams ( $A \boldsymbol{n}$ $1, A n$ 2, $A n$ 3) and one crocodile (An 4). Their spectra were compared with those of two pure bitumen samples: a fragment of natural asphalt from the Dead Sea (Ref 1), and a commercial powder of bitumen of Judea ( $\boldsymbol{R} \boldsymbol{e f} 2)$.

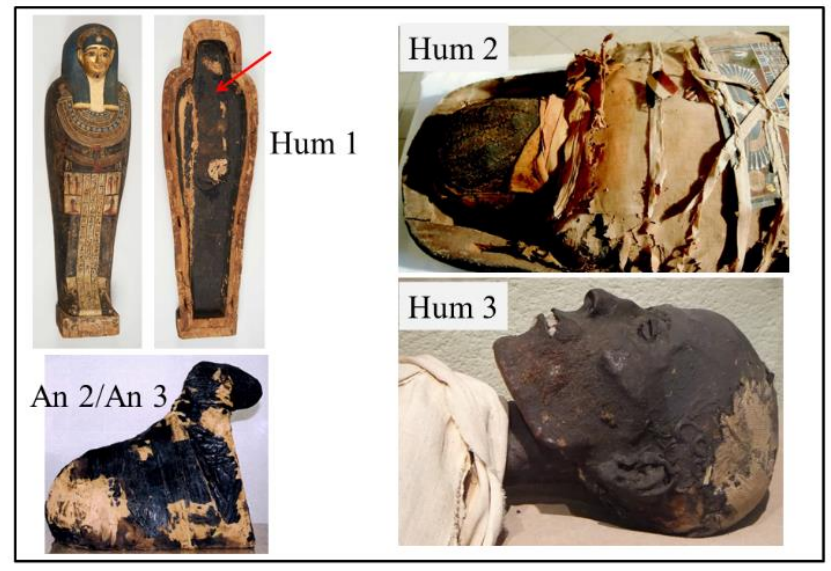

Figure 1. Origin of some samples of black matters studied by EPR; Hum 1) Coffin of Irethorerou (Ptolemaïc dynasty), The Art and History Museum, Narbonne, France, the black matter was sampled at the bottom of the coffin (arrow), reproduced with permission of C2RMF/Anne Chauvet; Hum 2) Human mummy of the Late Period, The Hieron museum, Paray-le-Monial, France, the sample is a fragment of black matter covering the mummy, reproduced with permission of C2RMF/Hélène Guichard; Hum 3) Head of the mummy from the Late period, Chateau-musée, Boulogne, France, the sample was taken from the mummy's neck, reproduced with permission from Frédérique Vincent; An 2/An 3) Ram mummy of the Late Period, The Thomas Dobrée museum, Nantes, France, $A n \mathbf{2}$ and $\boldsymbol{A n} \mathbf{3}$ samples are a fragment of black matter and a fragment of tissue with brown matter, respectively, reproduced with permission of C2RMF.

Table 1: Origin of the samples of black matters

\begin{tabular}{|cl|}
\hline Label & \multicolumn{1}{c|}{ Origin } \\
\hline Hum 1 & $\begin{array}{l}\text { Anthropomorphic coffin, upper Egypt, Ptolemaïc period (332 BC-30 BC). Black coating in the bottom } \\
\text { of the coffin }\end{array}$ \\
Hum 2 & Human mummy, Egypt, late Period (end of the $\mathrm{IV}^{\text {th }}$ century BC); black coating covering the mummy \\
Hum 3 & $\begin{array}{l}\text { Human mummy, Egypt, Late Period (XXI }{ }^{\text {th }}-\mathrm{XXV}^{\text {th }} \text { dynasty). Black matter taken from the neck of the } \\
\text { mummy. }\end{array}$ \\
An 1 & Ram mummy, Upper Egypt, Late Period; $672 \mathrm{BC}-322 \mathrm{BC}$; black coating covering the mummy. \\
An 2 & Ram mummy, Upper Egypt, Late Period (664 BC-332 BC); black coating covering the mummy \\
An 3 & Ram mummy (the same as An 2); tissue with brown matter covering the mummy. \\
An 4 & Crocodile mummy, Upper Egypt, Ptolemaic period; black matter covering the skull. \\
Ref 1 & Fragments of natural asphalt from the Dead Sea \\
Ref 2 & Commercial powder of bitumen of Judea
\end{tabular}


EPR spectra. Examples of cw-EPR spectra at X-band and Q-band are shown in Fig.2, for the Dead Sea asphalt (Ref 1) and the black coatings of mummies An 2 and Hum 3. The other spectra are given in Supplementary Information Figs. S2, S3 and S4. All spectra show the two paramagnetic species classically present in bitumen and oil, ${ }^{17,20,31,32}$ and recently identified by $\mathrm{X}$-band EPR in black coatings of mummies: ${ }^{28}$ (i) organic radicals $\mathrm{C}^{0}$ of asphaltene, represented by a single and intense line in the central part of the spectrum, with $g=1.9994$, and (ii) the classical spectrum of vanadyl porphyrin (VO-P) complexes embedded in the asphaltene, resulting from the hyperfine (hf) interaction of the $S=1 / 2$ electron spin of $\mathrm{V}^{4+}$ (configuration $3 \mathrm{~d}^{1}$ ) with the $I=7 / 2$ nuclear spin of the $100 \%$ abundant ${ }^{51} \mathrm{~V}$ isotope. This hf interaction gives two sets of $2 I+1=8$ lines characterized by $g$ and hf parameters $A$ given in Table 2. Parameters $g_{/ /}$and $A / /$ correspond to VO-P complexes with the V-O bond oriented along the external field $\mathbf{B}_{0}$. The most intense central set of 8 lines, with parameters $g_{\perp}$ and $A_{\perp}$ corresponds to VO-P complexes oriented with $\mathbf{B}_{0}$ lying in the porphyrin plane (and thus perpendicular to the $\mathrm{VO}$ bond). The ${ }^{51} \mathrm{~V}$ hf lines probed in HYSCORE (at X-band) and ENDOR (at Q-band) experiments are represented by arrows in Fig.2a and 2b, respectively.

In addition, the black coatings of $\boldsymbol{A n} 2$ (Fig.2), Hum 1, 2 and $\boldsymbol{A n}$ 1, 3, 4 samples exhibit additional lines (some of them are marked by green circles in Fig.2 and Figs. S2 and S3) characterized by $g_{/ /}=1.925 \pm 0.003, g_{\perp}=1.978 \pm 0.003, A_{/ /}=176( \pm 3) \times 10^{-4} \mathrm{~cm}^{-1}$ and $A_{\perp}=$ $70 \pm(3) \times 10^{-4} \mathrm{~cm}^{-1}$ and attributed to non-porphyrinic vanadyl complexes with oxygenated ligands (referred to as VO-nP complexes). ${ }^{28}$ These complexes are thought to result from the interaction of bitumen with other bioorganic substances (resins, wax, fat ...) of the black matters. The only exception is Hum 3 sample (the human mummy from the Boulogne museum, Fig.2), which exhibit only the VO-P spectrum, like pure bitumen ( $\boldsymbol{R}$ ef 1 and $\boldsymbol{R} \boldsymbol{e f} 2)$. We concluded that mummy Hum 3 was covered with pure bitumen, which was confirmed by GC-MS analysis. ${ }^{28}$ In addition, the spectrum of $\boldsymbol{H u m ~ 3 , A n ~} 1$ (Fig. S3) and, to a lesser extent $\boldsymbol{A n}$ 2, show a broad baseline distortion due to a ferromagnetic resonance (FMR) signal of iron oxide microparticles. ${ }^{28}$ This FMR signal, which does not give electron spin echo, can be eliminated by recording the echo-detected EPR spectrum (ED-EPR), as clearly shown for $\boldsymbol{A n} \mathbf{2}$ and $\boldsymbol{H u m}$ 3 at X-band (Fig. 2a). 


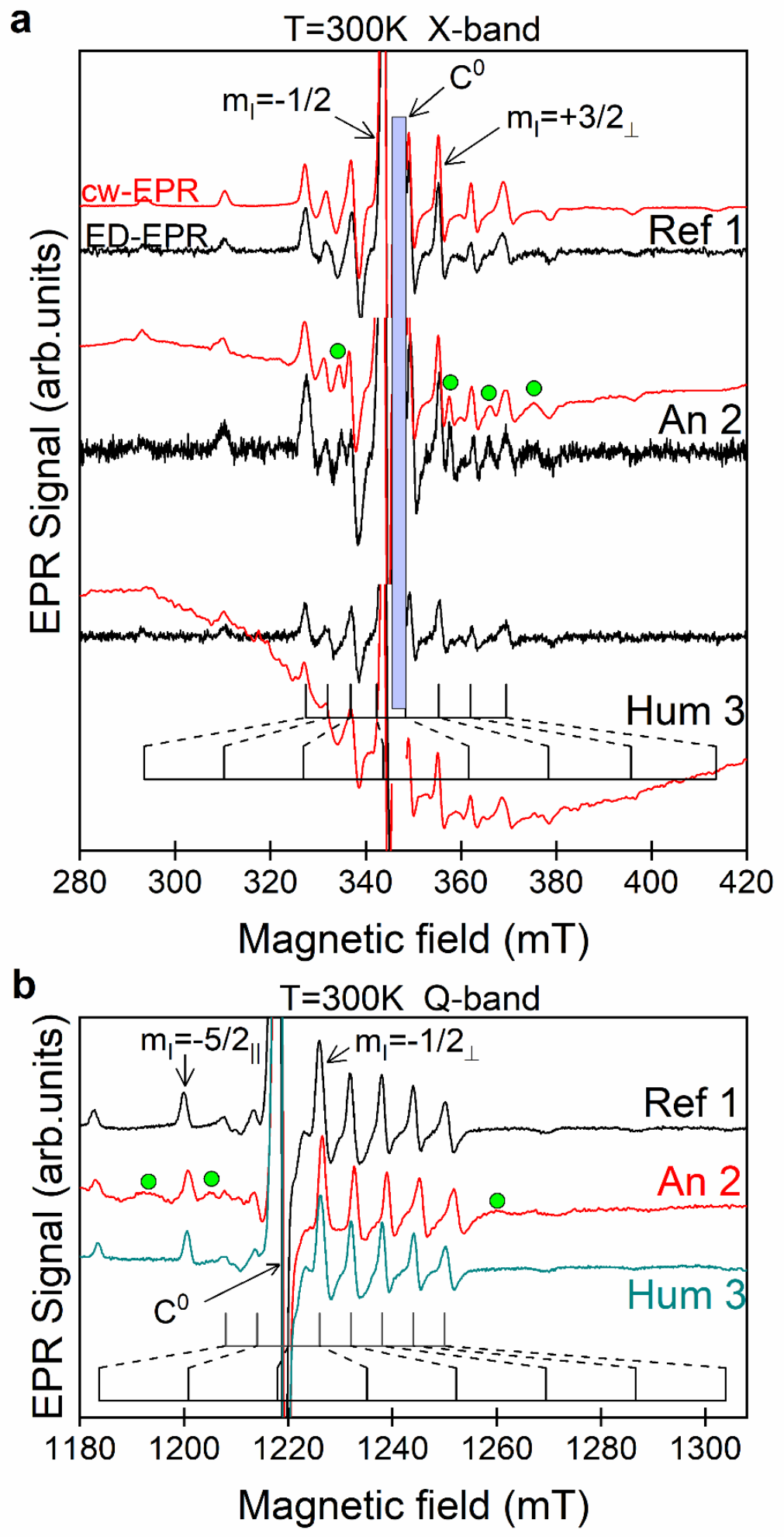

Figure 2. EPR spectra at room temperature of the black matters from samples Ref 1 , An 2 and $\mathrm{Hum} \mathrm{3;}$ a) cw-EPR spectra (in red) and pseudo-modulated ED-EPR spectra (in black) at X-band; the blue area represents the position of the strong $\mathrm{C}^{0}$ signal, that has been suppressed for the sake of clarity; b) cw-EPR spectra at Q-band; some EPR lines of VO-nP complexes in An 2 are represented by green circles. The magnetic field settings for ENDOR and HYSCORE experiments correspond to ${ }^{51} \mathrm{~V}$ hyperfine lines marked by arrows. 
Table 2: EPR parameters of VO-P complexes in Ref 1, An 2 and Hum 3 samples

\begin{tabular}{lll}
\hline Sample & $g$-factors $( \pm 0.002)$ & $A\left(\right.$ in $\left.10^{-4} \mathrm{~cm}^{-1}\right)$ \\
\hline Ref 1 & $g_{/ /}=1.959$ & $A_{/ /}=157 \pm 3$ \\
& $g_{\perp}=1.980$ & $A_{\perp}=54 \pm 2$ \\
\hline An 2 & $g_{/ /}=1.956$ & $A_{/ /}=158 \pm 3$ \\
& $g_{\perp}=1.977$ & $A_{\perp}=55 \pm 2$ \\
\hline Hum 3 & $g_{/ /}=1.957$ & $A_{/ /}=155 \pm 3$ \\
& $g_{\perp}=1.978$ & $A_{\perp}=52 \pm 2$ \\
\hline
\end{tabular}

The EPR parameters of VO-P complexes were deduced from the fitting of spectra at both $\mathrm{X}$ and Q-bands (Table 2). The slight differences of EPR parameters between the three samples fall within error bars of the simulations, except for parameter $A_{\perp}$, for which the differences are clearly visible on the spectra at Q-band (Fig. 2b).

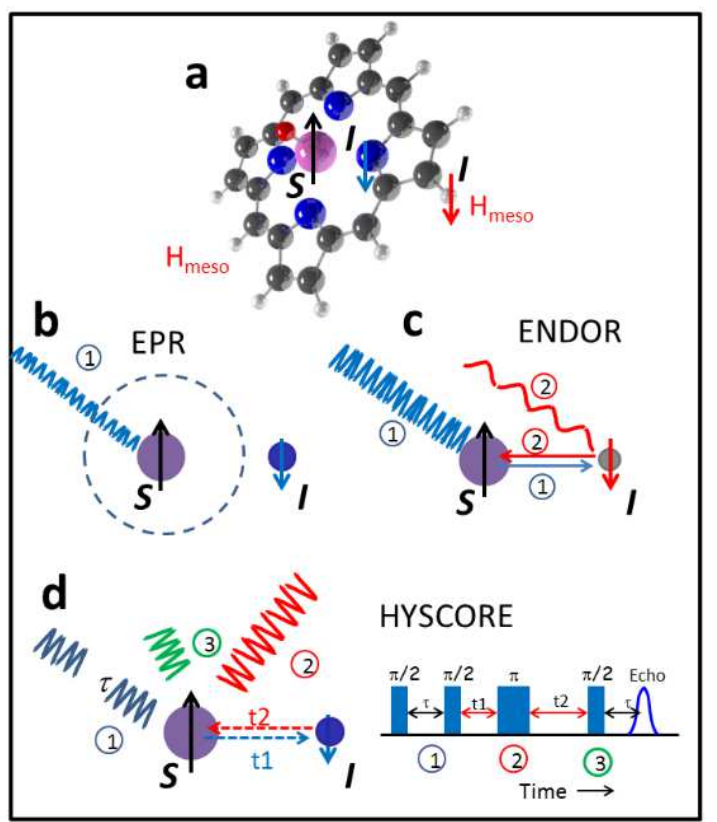

Figure 3. Principle of cw-ENDOR and HYSCORE spectroscopy; a) Schematic structure of a porphyrin-vanadyl complex, showing the electron spin $S$ on vanadium and two nuclear spins $I$ on nitrogen and on the bridging hydrogen $\mathrm{H}_{\text {meso }}$; b) EPR with a non-saturating microwave field, the dashed circle representing the limited resolution of EPR, which does not reach neighboring ligand nuclei; c) cw-ENDOR: a saturating microwave field modifies the populations of the nuclear spin state (step 1), while a strong rf field at nuclear frequency restores the nuclear populations, which in turn desaturates the EPR transition (step 2); d) HYSCORE spectroscopy: a sequence of two $\pi / 2$ microwave pulses separated by time $\tau$ induces a nuclear coherence in each electron spin state $m_{s}$ (step 1 ), a $\pi$ pulse after time $t 1$ produces a transfer of nuclear coherence between the two electron spin states $m_{s}$ (step 2); after an evolution time $\mathrm{t} 2$, the nuclear coherences are transferred back to the electron coherence by a $\pi / 2$ pulse, giving an electron spin echo at time $\tau(\operatorname{step} 3)$. 
The shape of EPR spectra of VO-P complexes (Fig.2) is entirely controlled by the anisotropy of the $g$-factor and of the strong hf interaction with the central ${ }^{51} \mathrm{~V}$ nucleus, which reflect the electronic structure and the geometry of the complex. For this reason, the weak unresolved hf interactions with ${ }^{1} \mathrm{H},{ }^{14} \mathrm{~N}$ and ${ }^{13} \mathrm{C}$ nuclei of the porphyrin ligand can only be revealed by ENDOR and HYSCORE spectroscopy.

${ }^{1}$ H-ENDOR analysis. In a cw-ENDOR experiment, a specific EPR transition is partially saturated at high microwave power and at fixed magnetic field, which modify the population of the nuclear spin states. A saturating radiofrequency (rf) field of frequency $v$ is then swept through the NMR frequencies of ${ }^{1} \mathrm{H}$ nuclei. The populations of nuclear spin states are modified at each nuclear resonance frequency, which are detected by a change in the EPR intensity (Fig.3c). A typical ${ }^{1} \mathrm{H}$-ENDOR spectrum at Q-band of VO-P complex in pure bitumen $\boldsymbol{R e f} \mathbf{2}$ is shown in Fig.4a, recorded at observing fields corresponding to the $m_{I}=-1 / 2 \perp$ and $-5 / 2 / /{ }^{51} \mathrm{~V}$-hf lines (arrows in Fig.3b). The ${ }^{1} \mathrm{H}$ signal is centered on the Larmor frequency $v_{\mathrm{H}}$ of hydrogen (typically $v_{\mathrm{H}}=51.9 \mathrm{MHz}$ for a magnetic field $1226 \mathrm{mT}$ ). As ENDOR spectra are recorded as first derivative of the ENDOR enhancement, the spectral features occur at angular turning points characterized by $v_{-} v_{\mathrm{H}}= \pm A / / 2$ and $v_{-v_{\mathrm{H}}}= \pm A_{\perp} / 2$, where $A_{/ /}\left(A_{\perp}\right)$ correspond to the $\mathrm{hf}$ parameters of hydrogen atoms in VO-P complexes oriented such that the external field $\mathbf{B}_{0}$ is parallel (perpendicular) to the V...H directions. The shape and parameters of the ENDOR spectrum depends on the nature of the $\mathrm{CH}$ bond, on the V...H distance, and on the set of molecular orientations selected by the magnetic field settings. For an observing field set at the $-5 / 2 / /$ transition of VO-P, this corresponds to the selection of vanadyl complexes oriented with the VO bond nearly parallel to the field vector $\mathbf{B}_{0}$, which is thus perpendicular to all the $\mathrm{V} \ldots \mathrm{H}_{\text {meso }}$ directions of the porphyrin plane (see Fig.2a) so that only two ENDOR lines corresponding to $A_{\perp}$ are observed for these hydrogens. Alternatively, for a field setting at the $1 / 2_{\perp}$ transition of VO-P, the selected vanadyl complexes correspond to $\mathbf{B}_{0}$ lying in the porphyrin plane and thus covering all possible angles with $\mathrm{V}$.... $\mathrm{H}_{\text {meso }}$ directions, so that both $A_{/ /}$and $A_{\perp}$ ENDOR lines are observed in this case (Fig.4a). The values $A_{/ /}=2.6 \mathrm{MHz}$ and $A_{\perp}=0.3 \mathrm{MHz}$ measured for VO-P correspond to the values known for the hydrogen $\mathrm{H}_{\text {meso }}$ of porphyrin in vanadyl porpyrin complexes. ${ }^{33,34}$ 


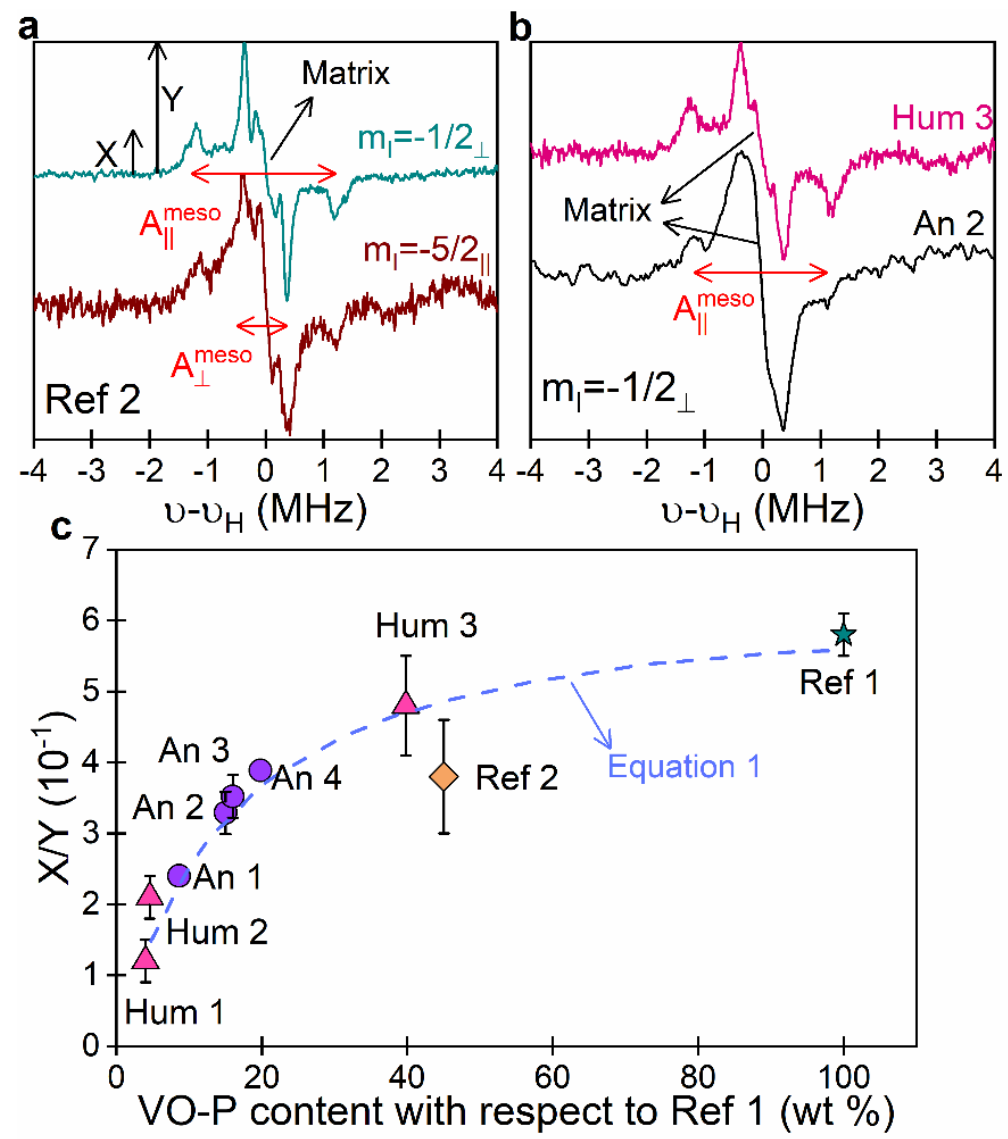

Figure 4. ${ }^{1} \mathrm{H}-\mathrm{ENDOR}$ at Q-band and at $100 \mathrm{~K}$ of VO-P complexes. a) bitumen of Judea (Ref 2), recorded by observing the $m_{I}=-1 / 2 \perp$ and $-5 / 2 / /$ hf lines of ${ }^{51} \mathrm{~V}$ (shown in Fig.2b); b) Hum 3 and An 2 samples recorded at the $m_{I}=-1 / 2 \perp$ hf position; c) Variation of the ratio X/Y of ENDOR amplitudes versus the weight-normalized VO-P content of samples; The interrupted line was calculated from Eq. 1 with $b=15$.

It must be noticed that additional ENDOR features that would be expected at $A / /=1.3 \mathrm{MHz}$ and $A_{\perp}=0.4 \mathrm{MHz}$ for hydrogen of pyrrole groups, ${ }^{34,35}$ are not observed in our ENDOR spectra. This indicates that all pyrrolic hydrogen atoms are substituted with alkyl groups, as known in the case of vanadyl geoporphyrins in oil and bitumen, such as vanadyl etioporphyrin (VO-EP) and vanadyl deoxophylloerythroetioporphyrin (VO-DPEP) for example (Fig. S6) ${ }^{36}$ The narrow signal centered at $v_{\mathrm{H}}$, referred to as the "matrix" line, ${ }^{37}$ corresponds to more distant hydrogen atoms, which are weakly coupled to the electron spin by dipolar interactions. These include hydrogens of the alkyl side groups linked to pyrroles (mainly methyl and ethyl groups, see Fig. S6), to hydrogens of the polyaromatic molecules of the surrounding asphaltene molecules, and to hydrogens of the bioorganic molecules (resins, waxes and fats) of the black matters. In the case of pure bitumen ( $\operatorname{Ref} 1$ and $\operatorname{Ref} 2$, see Fig.4a and Fig. S5), only the alkyl side groups of porphyrin ligands and the asphaltene hydrogens can contribute to the weak matrix ENDOR line. 
Although EPR spectra of VO-P complexes are very similar in bitumen and all black coating samples (Figs. 2b and S4), ${ }^{28}$ their ENDOR spectra are somewhat different. The $A_{/ /}$lines of $\mathrm{H}_{\text {meso }}$ atoms are well resolved in pure bitumen $\operatorname{Ref} 1, \operatorname{Ref} 2$ and in the human mummy $\mathbf{H u m} 3$ (Fig.4b) while they broaden and decrease in intensity in black coatings of all other mummies (Figs.4b and S5). Moreover, a pronounced matrix ENDOR signal centered at $v_{\mathrm{H}}$ appears in all samples except pure bitumen (Ref 1 and 2) and Hum 3 (Figs.4b and S5).

This variation of the ${ }^{1} \mathrm{H}-\mathrm{ENDOR}$ spectrum can be quantified by the ratio $\mathrm{X} / \mathrm{Y}$, where $\mathrm{X}$ is the amplitude of the spectrum at the position of the $A_{/ /}$line, and $\mathrm{Y}$ the amplitude at the position of the $A_{\perp}$ line, including the matrix line (see Fig. 4a). The variation of X/Y with VO-P content of the black coatings, normalized to the same mass of Dead Sea bitumen $(\boldsymbol{R} \boldsymbol{e f} \boldsymbol{1})$ is shown in Fig. 4c. We observed a regular decrease of $\mathrm{X} / \mathrm{Y}$ with decreasing content of VO-P per unit mass of black matter, the VO-P content being related to the bitumen content. A value $\mathrm{X} / \mathrm{Y} \approx 5.6$ is found for non-degraded Dead Sea bitumen (Ref 1), where the environment of VO-P consists mostly in asphaltene. Mixing bitumen with increasing amounts of natural substances has three effects: (i) the decrease of VO-P content per unit mass of black matter (i.e the decrease of EPR intensity of VO-P); (ii) an increase of the matrix ENDOR line (i.e an increase of Y) due to increasing amounts of hydrogen atoms of bioorganic molecules in the vicinity of VO-P, and; (iii) a broadening and weakening of the $\mathrm{A} / / \mathrm{ENDOR}$ lines of hydrogen atoms $\mathrm{H}_{\text {meso }}$ (i.e. a decrease of their amplitude $\mathrm{X}$ ) resulting from a disorder (distribution of hf interaction values $A / /$ ) in the structure and environment of VO-P complexes. However, this disorder effect is too low to modify the global square planar geometry of VO-P, which has no visible effect on the shape and parameters of EPR spectra. The reference bitumen $\boldsymbol{R} \boldsymbol{e f} \mathbf{2}$ deviates slightly from the general trend in Fig.4c. However, since this Judea bitumen is commercial, it may have undergone some undocumented treatment that could have modified some of its characteristics.

It is important to note that a matrix ENDOR line can occur only if the vanadium-proton distances $R$ are sufficiently small to give non-zero electron-proton dipolar interactions (i.e $R<$ $5-6 \mathrm{~nm}) .{ }^{37,38}$ We may conclude that only protons at distance $R>0.6-0.7 \mathrm{~nm}$ from the vanadium atom ( half the size of the porphyrin ligand) and $R<5-6 \mathrm{~nm}$ (limit for non-zero dipolar interaction) can contribute to the ${ }^{1} \mathrm{H}$ matrix line. This limited distance range for matrix protons has two consequences: (i) for a given proportion of bitumen and natural substances, the amplitude $Y$ of the matrix line should increase ( $X / Y$ decrease) upon decreasing the radius $R_{A}$ of bitumen aggregates in the black matters (see Fig.S7 for a schematic representation), and reach a maximum amplitude for $R_{A}<5-6 \mathrm{~nm}$ (i.e. all VO-P complexes "see" protons of natural 
substances); (ii) for bitumen aggregates with mean $R_{A}$ value, $X / Y$ should decrease upon increasing content of natural substances, as more and more bioorganic hydrogen atoms are present in the vicinity of VO-P complexes. According to (i), $X / Y$ should be nearly independent of VO-P content of the black matter if $R_{A}$ lies in the micrometer range or larger, because in this case, only the small fraction of VO-P close to the surface of bitumen aggregates "see" the bioorganic protons. According to (i) and (ii), the fact that the decrease of $X / Y$ with decreasing VO-P content is regular (Fig. 4c) for our corpus of black coatings (if we except the commercial bitumen $\boldsymbol{R}$ ef 2) suggests that $R_{A}<6-7 \mathrm{~nm}$ in all cases, and that $X / Y$ depends only on the ratio bitumen/natural substances of the black matters.

A lower limit of the size $R_{A}$ of bitumen aggregates can also be estimated from the fact that VO$\mathrm{P}$ and radicals $\mathrm{C}^{0}$ are spatially connected in asphaltene, with (VO-P)- $\mathrm{C}^{0}$ distances not larger than 1-3 nm. ${ }^{39}$ We previously showed that such spatial connection is conserved in bitumen of Egyptian black coatings. ${ }^{28}$ Consequently, we may roughly estimate that the sizes of bitumen aggregates in the studied black coatings lie in the range $\sim 1 \mathrm{~nm}<R_{A}<6-7 \mathrm{~nm}$. Three scales of asphaltene aggregation were proposed in the Yen-Mullins model of asphaltene hierarchical structure: ${ }^{40}$ the molecular $(\sim 1.5 \mathrm{~nm})$, the nanocluster $(\sim 2.0 \mathrm{~nm})$ and the Cluster $(\sim 5.0 \mathrm{~nm})$ scales. It appears that the sizes of bitumen aggregates in black coatings correspond to the cluster scale of the Yen-Mullins model. ${ }^{40}$ Whatever the actual distribution size of asphaltene aggregates be, this ENDOR analysis shows that the bitumen and natural substances are intimately mixed in black coatings. For such small sizes of bitumen aggregates, where the surface/volume ratio is high, this could also explain why a significant fraction of VO-P are transformed into oxygenated VO-nP complexes at interfaces between bitumen aggregates and natural substances. ${ }^{28}$

The regular variation of the ENDOR shape factor $\mathrm{X} / \mathrm{Y}$ with VO-P content (Fig.4c) can be reproduced with a simple model considering that $X$ and $Y$ amplitudes are both sums of contributions from protons of VO-P complexes and of the matrix layer around the bitumen aggregates, respectively, so that the ratio $X / Y$ is given by (see SI for demonstration):

$$
\frac{X}{Y}=\frac{X_{V O P}}{Y_{V O P}} \times \frac{x+0.048 \times b}{x+b}
$$

where $x$ is the VOP content with respect to $\operatorname{Ref} \boldsymbol{1}$ (the abscissa in Fig. 4c), $X_{V O P}$ and $Y_{V O P}$ are the peak-heights of the parallel and perpendicular components of the ${ }^{1} \mathrm{H}$ ENDOR spectrum of an isolated VO-P complex (measured in $\operatorname{Ref} \boldsymbol{1}$ for $m_{I}=-1 / 2_{\perp}$ ). Parameter $b$ in Eq. 1 is given by $b=a Y_{m} / Y_{V O P}$, with $Y_{m}$ the peak-to-peak half-height of the ENDOR line of a matrix proton. The 
ratio $Y_{m} / Y_{V O P}$ depends only on the ENDOR lineshapes. Parameter $a$ is a function of the bitumen content of the black matters and of the mean size of bitumen aggregates (see SI for more details). As $X_{V O P} / Y_{V O P} \approx 0.625$ for $\boldsymbol{R e f} 1$, there is only one adjustable parameter $b$ in Eq.1. Experimental data were nicely fitted to Eq.1 with $b=15$ (Fig.4c). This good agreement shows that $a$ is a constant almost independent of sample. As developed in SI, this means that the larger the concentration of bitumen in the black matters, the larger the mean size of bitumen aggregates. Consequently, this result shows that a simple measurement of the amplitude of ENDOR lines can give direct information on the bitumen content of a black coating.

${ }^{14} \mathrm{~N}$-HYSCORE analysis. The very small variations of EPR parameters $g$ and $A$ of VO-P from one sample to another (Table 2) may suggest that several types of slightly different VO-P complexes are present in variable proportions in the bitumen component of black coatings. We used HYSCORE spectroscopy at X-band to discriminate different types of VO-P by their ${ }^{14} \mathrm{~N}$ $\mathrm{hf}$ interaction, with the perspective to use in the future these metallic complexes for getting information on the geographical origin of the bitumen and on its chemical or thermal treatment during the preparation of the mummy. In pulsed EPR spectroscopy, ${ }^{29}$ a spin echo is generated by a series of $\pi / 2$ and $\pi$ microwave pulses ( $\pi$ and $\pi / 2$ represent the rotation angles of the electron spin magnetization) separated by controlled time delays (Fig.3d). By varying these time delays, the echo intensity is modulated at frequencies of the hf interactions. HYSCORE spectroscopy is based on the pulse sequence $\pi / 2-\tau-\pi / 2-\mathrm{t} 1-\pi-\mathrm{t} 2-\pi / 2-\tau$-echo, where $\tau$ is the delay between the first and second $\pi / 2$ pulses. The first $\pi / 2$ pulse generates an electronic coherence (a mixing of the two $m_{s}= \pm 1 / 2$ states), and the second $\pi / 2$ pulse after time $\tau$ transfers the electronic coherence to nuclear coherences (mixing of $m_{I}$ states). After an evolution time t1, a $\pi$ pulse transfers the nuclear coherence from one $m_{s}$ state to the nuclear coherences of the other $m_{s}$ state, which creates correlations between nuclear transitions of these two $m_{s}$ states. After another evolution time $\mathrm{t} 2$, a third $\pi / 2$ pulse transfers the nuclear coherence back to the electronic coherence for detection, which generates an electron spin echo after time $\tau$. The echo intensity is measured for the two times $t 1$ and $t 2$, which are varied stepwise at constant $\tau$ value. The $2 \mathrm{D}$ frequency plot (HYSCORE) is obtained by 2D-Fourier transformation of the data set in time domain.

The correlations between nuclear transitions in the two $m_{s}= \pm 1 / 2$ states appear as cross peaks in the 2D frequency plot, which are distributed in two different quadrants $(+,+)$ and $(+,-)$ corresponding to $\omega_{2}>0, \omega_{1}>0$ and $\omega_{2}>0, \omega_{1}<0$, respectively. ${ }^{29}$ For an electron spin $S=1 / 2$ 
interacting with an $I=1 / 2$ nuclear spin such as ${ }^{13} \mathrm{C}$ and ${ }^{1} \mathrm{H}$, we expect two cross peaks in each quadrant, which take the shape of ridges perpendicular to the diagonal $\omega_{1}=\omega_{2}$ for anisotropic hf interactions in disordered materials. ${ }^{29}$ The spectrum is more complicated for a nuclear spin $I$ $=1$ such as ${ }^{14} \mathrm{~N}$, which can give up to 18 cross peaks and ridges in each quadrant. ${ }^{41}$ The situation is even more puzzling if the electron spin is coupled with several nuclear spins (which indeed is the case of VO-P) because such multi-spin systems can give additional zero- and multi-quanta coherences, as well as suppression effects. ${ }^{38}$ Fortunately, many of these spectral features are too weak to appear in the 2D-plot, so that the HYSCORE spectra remain interpretable. ${ }^{42-44}$ Representative HYSCORE spectra for samples Ref $\mathbf{1}, \mathbf{A n} \mathbf{2}$ and Hum 3 in the frequency range 0 to $\pm 12 \mathrm{MHz}$ are shown in Fig.5. HYSCORE spectrum of Ref 2 is reported in Fig. S8. We could not obtain acceptable HYSCORE spectra of Hum 1, Hum 2 and An 1 because of their low amount of VO-P. The spectra were recorded from two field settings in the EPR spectra (arrows in Fig.2a): the $m_{I}=+3 / 2_{\perp}$ hf line (Fig.5b) which select VO-P complexes oriented with their VO axis perpendicular to the external field $\mathbf{B}_{0}$ (i.e $\mathbf{B}_{0}$ lies in the porphyrin plane), and the strong $m_{I}=-1 / 2 \mathrm{hf}$ line (Fig.5a) whose position is almost independent on the field orientation (no selection of molecular orientations). Since our goal was to explore the diversity of VO-P complexes, we focused the spectral analysis on the narrow and relatively intense correlation peaks highlighted by the black boxes in the $(+,-)$ quadrant of Fig.5, which are represented in more details in Fig.6 for Ref 1, Hum 3 and An 2, and in Fig.S8 for Ref 2.

The energy level diagram in Fig. 6 describes the spin states and the corresponding nuclear transitions for an $S=1 / 2, I=1$ system. The frequencies for the single quantum $\left(\Delta m_{I}=1\right)$ and double quantum $\left(\Delta m_{I}=2\right)$ transitions, referred to as sq and dq transitions, respectively, are given by: 42,44

$v_{1 s q}^{ \pm}=\frac{A}{2} \pm v_{N}+\frac{3 Q}{2}+\left(2^{\text {nd }}\right.$ order terms $)$

$v_{2}^{ \pm} s q=\frac{A}{2} \pm v_{N}-\frac{3 Q}{2}+\left(2^{\text {nd }}\right.$ order terms $)$

$v_{d q}^{ \pm}=A \pm 2 v_{N}+\frac{\mathrm{A}^{(2)}}{(A / 2) \pm v_{N}}$

where $v_{N}$ is the nuclear Zeeman frequency, and $A$ and $Q$ the hf interaction and the quadrupolar interaction, respectively, of ${ }^{14} \mathrm{~N}$ nuclei for a given field orientation. The full expressions including second order terms in $v_{s q}$ and their estimation are given in SI. The widths and positions of $\mathrm{dq}$ transitions (Eq.3) are determined by the small anisotropy of the ${ }^{14} \mathrm{~N}-\mathrm{hf}$ 
interaction $A$ and by the $2^{\text {nd }}$ order correction, while the widths and positions of sq transitions (Eqs.2) are mostly controlled by the strong anisotropy of $Q$ (which is a traceless tensor) in addition to $A$ and $2^{\text {nd }}$ order corrections. For this reason, the sharp dq-dq correlation peaks, which depend only on $A$ to first order, have sufficiently high resolution and sensitivity to be used for the identification of various VO-P complexes. The quadrupolar interaction $Q$ for each type of VO-P can only be determined by using sq frequencies (Eqs.2).
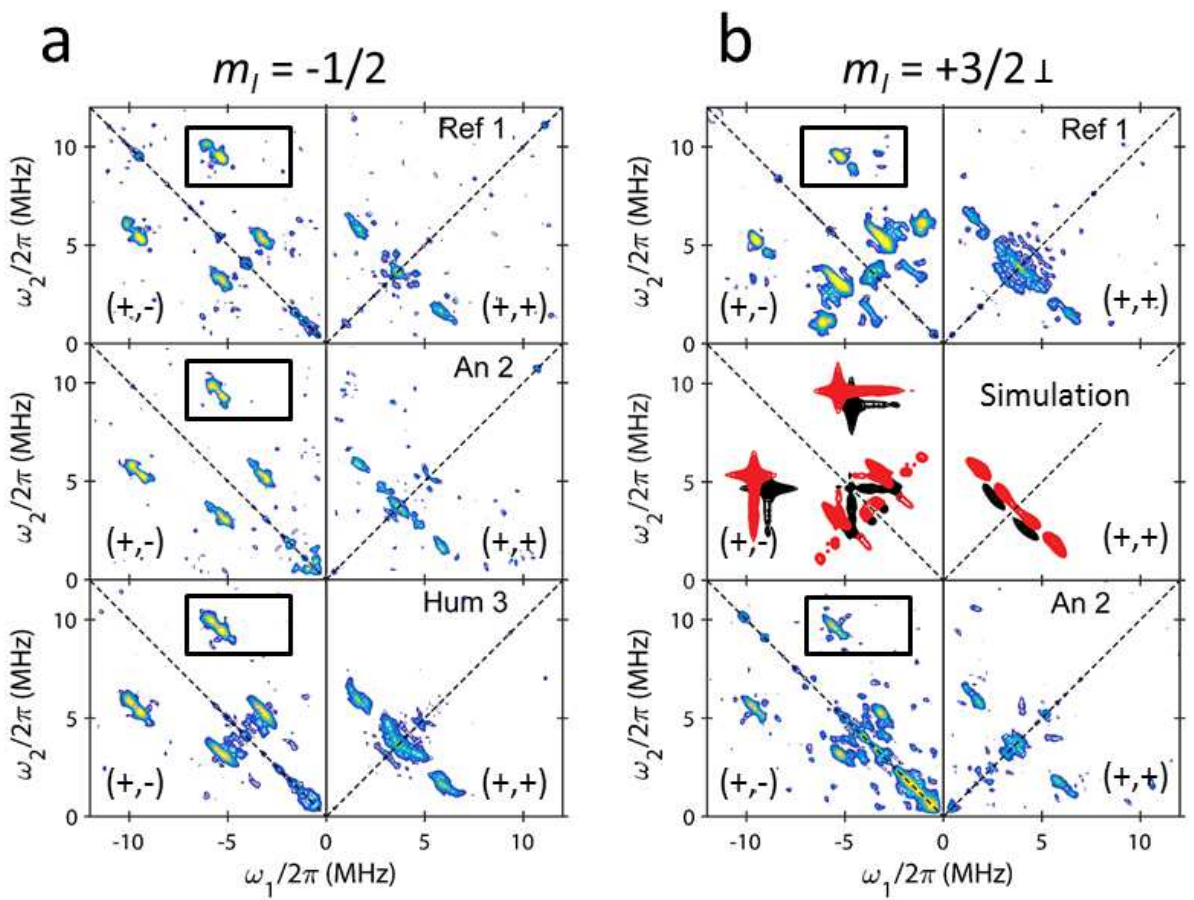

Figure 5. HYSCORE spectra at X-band and at $6 \mathrm{~K}$ of VO-P in Dead Sea asphalt Ref 1 and in black coating of An 2 and Hum 3 mummies, recorded by observing a) the $m_{I}=-1 / 2$ and b) the $m_{I}=+3 / 2 \perp$ hf lines marked by arrow in Fig.2a. The correlation peaks described in more details in Fig. 6 are delimited in rectangular boxes. Simulated spectra of VO-P1 (in red) and VO-P2 (in black) in the middle of panel b.

Let us take the example of the HYSCORE of Dead Sea asphalt $\boldsymbol{R} \boldsymbol{e f} \mathbf{1}$, observed at the $m_{I}=$ $+3 / 2_{\perp}$ field position, and shown in the top right of Fig.6. Two distinct dq-dq peaks are clearly observed, representing two different types of VO-P complexes, hereafter referred to as VO-P1 and VO-P2. The frequency coordinates of these dq-dq peak are $[-5.3 ;+9.5] \mathrm{MHz}$ for VO-P1 and $[-4.6 ;+8.9] \mathrm{MHz}$ for VO-P2, with an error bar of about $\pm 0.1 \mathrm{MHz}$. The hf interaction $A$ is directly obtained from Eq.3: 


$$
A=\frac{2 v_{N}\left(v_{d q}^{+}+v_{d q}^{-}\right)}{8 v_{N}-\left(v_{d q}^{+}-v_{d q}^{-}\right)}
$$

where the $2^{\text {nd }}$ order term of Eq.3 is naturally eliminated. With $v_{N}=1.1 \mathrm{MHz}$ at $355.7 \mathrm{mT}$, we obtained $A=-7.28 \mathrm{MHz}$ for VO-P1 and $A=-6.6 \mathrm{MHz}$ for VO-P2. The hf coupling was chosen negative on the basis of DFT calculation on VO-P complex in crude oil. ${ }^{45}$
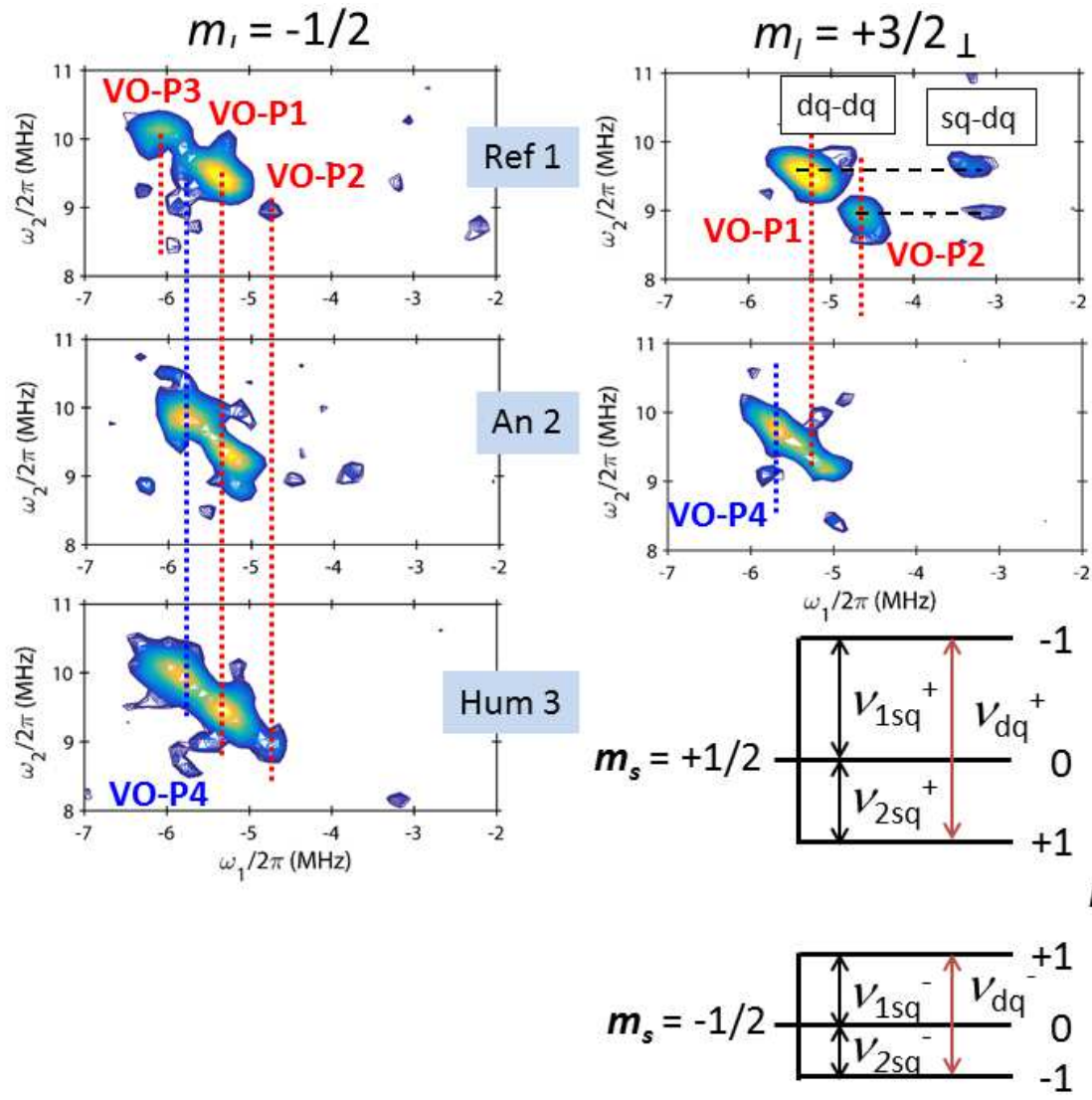

Figure 6. Portions of the HYSCORE spectra of Ref 1, An 2 and Hum 3 corresponding to dq-dq and dqsq correlation peaks, where $\mathrm{dq}$ and sq represent double quantum and single quantum transitions, respectively. The diagram represents the spin states and the different nuclear transitions for a $S=1 / 2, I=1$ spin system.

The vanadyl content of $\boldsymbol{R} \boldsymbol{e f} \mathbf{1}$ is sufficiently high to reveal two weaker sq-dq correlation peaks at $[-3.3 ;+9.5] \mathrm{MHz}$ for VO-P1 and $[-3.1 ;+8.9] \mathrm{MHz}$ for VO-P2, at the $m_{I}=+3 / 2 \perp$ field setting (Fig.6). As a sq-dq peak correlates a dq transition $v_{d q}$ of one $m_{s}$ state with a sq transition $v_{s q}$ of the other $m_{s}$ state, and considering Eqs.2 which give $v_{1 s q}^{+}-v_{1 s q}^{-} \approx v_{2 s q}^{+}-v_{2 s q}^{-} \approx 2 v_{N}$ to first order and $v_{1 s q}^{ \pm}+v_{2}^{ \pm} s q=v_{d q}^{ \pm}$, all sq and dq frequencies of the spin system in Fig.5 can be simply deduced. The results for VO-P1 and VO-P2 are shown in Fig.S9. Neglecting again $2^{\text {nd }}$ order 
terms, the quadrupolar parameter $Q$ can be estimated from Eqs.2 by: $Q \approx\left(v_{1 s q}^{-}-v_{2 s q}^{-}\right) / 3 \approx\left(v_{1 s q}^{+}-v_{2 s q}^{+}\right) / 3$. The values for VO-P1 and VO-P2 are $|Q| \approx 0.47 \pm 0.03$ $\mathrm{MHz}$ and $|Q| \approx 0.55 \pm 0.02 \mathrm{MHz}$, respectively (see SI for the estimation of the second order terms).

The ${ }^{14} \mathrm{~N}$-hf interaction is anisotropic, with two components $A_{/ /}$and $A_{\perp}$, corresponding to $\mathbf{B}_{0}$ parallel and perpendicular to the V-N bond. For HYSCORE spectra recorded from the $m_{I}=$ $+3 / 2_{\perp}$ field setting, which span all orientations of $\mathbf{B}_{\mathbf{0}}$ in the porphyrin plane, the measured value of $A$ is the average $\langle A\rangle_{\perp} \approx\left(A_{/ /}+A_{\perp}\right) / 2$, with $A_{/ /}=a_{i s o}+2 T$ and $A_{\perp}=a_{i s o}-T$. Parameters $a_{i s o}$ and $T$ are the isotropic and dipolar hf interactions, respectively. For the corresponding HYSCORE spectrum of $\boldsymbol{R e f} 1$ recorded with the $m_{I}=-1 / 2$ field setting (top left spectrum of Fig.6), where almost all molecular orientations are probed, the hf interaction measured from dq-dq peaks (Eq.4) is approximated as the average value over all the possible field orientations, given by $\langle A\rangle \approx\left(A_{/ /}+2 A_{\perp}\right) / 3$. The dq-dq peak of VO-P1 at $[-5.3 ;+9.5] \mathrm{MHz}$ gives $\langle A\rangle=-7.28 \mathrm{MHz}$ for this field setting. Combining the hf values $\langle A\rangle_{\perp}$ and $\langle A\rangle$ measured for the two observing fields shows that the hf interaction is mostly isotropic, with $a_{i s o}=-7.3 \mathrm{MHz}$ and $T<0.1 \mathrm{MHz}$ for VO$\mathrm{P} 1$. By the same procedure, the ${ }^{14} \mathrm{~N}$ parameters of VO-P2 are $a_{\text {iso }}=-6.5 \mathrm{MHz}$ and $T=0.2$ MHz. It is interesting to note that the value $a_{i s o}=-7.3 \mathrm{MHz}$ measured for VO-P1 is close to the value $a_{i s o}=-7.23 \mathrm{MHz}$ measured by pulsed-ENDOR for VO-P complexes in heavy crude oil from the Republic of Tatarstan (Russia), and the value $a_{i s o}=-7.2 \mathrm{MHz}$ measured by pulsedEPR for vanadyl octaethylporphyrin. ${ }^{45,46}$ This good concordance between measurements of different nature confirms that a simple measurement of the sharp dq-dq peaks recorded by observing the intense and nearly isotropic $m_{I}=-1 / 2$ EPR line, gives a good estimate of the isotropic hf coupling $\langle A\rangle \approx\left(A_{/ /}+2 A_{\perp}\right) / 3=a_{\text {iso }}$.

Vanadyl complex VO-P1 is present in the four studied samples, while VO-P2 complex is detectable only in Ref 1, Hum 3 and Ref 2 (Figs. 6 and S8). Another complex, referred to as

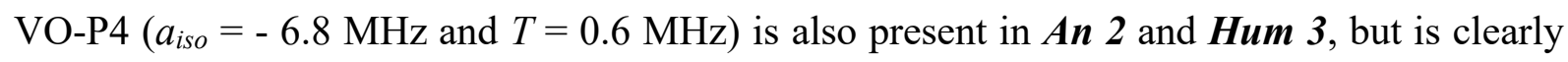
absent in $\boldsymbol{R} \boldsymbol{e} \boldsymbol{1}$ and $\boldsymbol{R} \boldsymbol{e f} \mathbf{2}$. However, an additional dq-dq peak attributed to a complex VO-P3 ( $a_{i s o} \approx-7.3 \mathrm{MHz}$ ), was detected only in pure bitumen samples $\boldsymbol{R} \boldsymbol{e f} \boldsymbol{1}$ and $\boldsymbol{R} \boldsymbol{e f} \boldsymbol{2}$ by observing the sharp $m_{I}=-1 / 2$ EPR transition. As sq-dq transitions could not be detected for VO-P3 and VO$\mathrm{P} 4$, it was not possible to give an estimation of $|Q|$ in these cases. The ${ }^{14} \mathrm{~N}$ parameters of the four VO-P complexes are reported in Table 3. 
Table 3: Experimental (exp) and simulated (sim) hyperfine and quadrupolar parameters for ${ }^{14} \mathrm{~N}$ nuclei $(\mathrm{nm}=$ not measured $)$ in VO-P complexes

\begin{tabular}{|llllll|}
\hline Complex & & $a_{i s o}(\mathrm{MHz})$ & $T(\mathrm{MHz})$ & $Q_{z z}(\mathrm{MHz})$ & Samples \\
\hline VO-P1 & Exp & -7.3 & $<0.1$ & 0.94 & Ref 1; Ref 2; Hum 3; An 2 \\
& sim & -7.3 & 0.1 & 1.0 & \\
VO-P2 & $\exp$ & -6.5 & 0.2 & 1.1 & Ref 1; Hum 2; Hum 3 \\
& $\operatorname{sim}$ & -6.6 & 0.2 & 1.0 & \\
VO-P3 & $\exp$ & $(\approx-7.3)$ & $n m$ & $n m$ & Ref 1; Ref 2 \\
VO-P4 & $\exp$ & -6.8 & 0.6 & $n m$ & Hum 3; An 2 \\
\hline
\end{tabular}

All this interpretation was based on the analysis of only a small portion of each HYSCORE spectrum (rectangular boxes in Fig.5). This procedure raises the question of the interpretation of all other correlation peaks and ridges present in the HYSCORE spectra (Fig. 5). To test the validity of the proposed analysis, the whole ${ }^{14} \mathrm{~N}-\mathrm{HYSCORE}$ spectra of VO-P1 and VO-P2 complexes recorded at the $m_{I}=+3 / 2_{\perp}$ field setting were simulated with Easyspin software, by adjusting the values of $a_{i s o}, T$ and $Q_{z z}$ (the $z$-component of the quadrupolar interaction). The result is shown in the middle of Fig.5b. Except for peak intensities, which are not correctly accounted for in the simulations, the main features of experimental HYSCORE spectra are well reproduced, taking into account the fact that the shape of the simulated spectra is very sensitive to the values of $a_{i s o}, T$ and $Q_{z z}$ parameters. This validation of nitrogen parameters reported in Table 3 calls for several comments:

(i) The simulated values $Q_{z z}=+0.9 \mathrm{MHz}$ and $+1.0 \mathrm{MHz}$ for VO-P1 and VO-P2 complexes are about twice the experimental values $|Q|=0.47 \mathrm{MHz}$ and $0.55 \mathrm{MHz}$ measured from sq-dq correlation peaks recorded at the $m_{I}=+3 / 2_{\perp}$ field setting. As the quadrupolar interaction is a traceless tensor (i.e. $Q_{z z}+Q_{x x}+Q_{y y}=0$ ), this means that the z-axis of the ${ }^{14} \mathrm{~N}$ quadrupolar interaction is perpendicular to the porphyrin plane, so that the experimental values measured here by setting the magnetic field in the porphyrin plane $\left(m_{I}=+3 / 2_{\perp}\right.$ EPR transition) correspond to $|Q| \approx Q_{x x} \approx Q_{y y} \approx Q_{z z} / 2$. 
(ii) All the other correlation peaks and ridges visible in the $(+,-)$ quadrant are clearly due to the same porphyrinic nitrogen atoms that give rise to the observed dq-dq peaks. Thus it is not necessary to invoke hf interactions with other nuclei $\left({ }^{14} \mathrm{~N},{ }^{13} \mathrm{C}\right.$ or other).

(iii) Unfortunately the $(+,+)$ quadrant does not give any information on the ${ }^{13} \mathrm{C} \mathrm{hf}$ interaction because the corresponding peaks are hindered under ${ }^{14} \mathrm{~N}$ correlations which come out in the same frequency range as ${ }^{13} \mathrm{C}\left(v_{C}=3.7 \mathrm{MHz}\right)$, as shown by the simulation in Fig. 5. Also, in a multi-spin system such as VO-P complexes, nuclei with weak modulations (such as ${ }^{13} \mathrm{C}$ and $\left.{ }^{1} \mathrm{H}\right)$ can be partially or totally suppressed by nuclei with deep modulations $\left({ }^{14} \mathrm{~N}\right),{ }^{38}$ explaining why we cannot measure ${ }^{13} \mathrm{C}$ hf interactions in VO-P complexes.

Focus on mummy Hum 3. This ${ }^{1} \mathrm{H}$ and ${ }^{14} \mathrm{~N}$ hf analysis of vanadyl probes in black coatings confirms the peculiarity of $\mathbf{H u m} 3$ compared to other samples of black matter, as previously evidenced by cw-EPR. ${ }^{28}$ Hum 3 was taken from the neck of the mummy found in Nehemsimontou's coffin (XXV ${ }^{\text {th }}$ dynasty, $744 \mathrm{BC}$ to $656 \mathrm{BC}$ ), purchased in 1837 by the museum of Boulogne (France) from a private collector. It later turned out that the mummy and the coffin had been assembled for the purpose of a better sale, a common practice in the $19^{\text {th }}$ century. This beautiful mummy is covered with a solid, black and shiny substance, and has therefore an unknown origin (Fig.1). Cw-EPR and GC-MS analysis showed that this black coating is made of pure bitumen. ${ }^{28}$ Contrary to other black coatings studied in this work (animal and human mummies, coffin), Hum 3 contains no VO-nP (non-porphyrinic vanadyl complexes), and its EPR spectrum is very similar to that of pure bitumen (Ref 1 and $\boldsymbol{R e f} 2){ }^{28}$ This similarity with native bitumen is confirmed by the almost identical ENDOR spectra of Hum 3, Ref 1 and $\boldsymbol{R e f} 2$ (Figs.4 and S5). These samples do not exhibit the intense ${ }^{1} \mathrm{H}$ matrix line present when natural substances are mixed with bitumen, which is consistent with the fact that $\mathbf{H u m} 3$ was made of pure bitumen.

Among the three vanadyl porphyrin complexes VO-P1, VO-P2 and VO-P3 detected in bitumen from the Dead Sea and Judea region (Ref 1 and $\boldsymbol{R e f} 2$ ), complexes VO-P1 and VO-P2 are also present in $\mathbf{H u m} 3$, while only VO-P1 was detected in $\boldsymbol{A n} \mathbf{2}$. As the latter contains less than 20\% of the VO-P content of $\boldsymbol{R}$ ef $1,{ }^{28}$ it is possible that VO-P2 peak intensity may be too low to be detected in this case. Bitumen from mummies $\boldsymbol{A n} \mathbf{2}$ and $\mathbf{H u m} \mathbf{3}$ contain also a fourth complex VO-P4, that is absent in reference bitumen $\boldsymbol{R} \boldsymbol{e f} \mathbf{1}$ and $\boldsymbol{R e f} 2$. Instead of VO-P4, $\boldsymbol{R} \boldsymbol{e f} \mathbf{1}$ and $\boldsymbol{R} \boldsymbol{e f} \boldsymbol{2}$ are characterized by the presence of VO-P3 complex. As the preparation of the embalming 
coating by ancient Egyptian implies that bitumen was heated to the liquid state in order to be mixed with the other ingredients and spread on the mummy, we may hypothesize that VO-P4 originates from the thermal transformation of an unstable complex VO-P3 initially present in bitumen. Laboratory experiments will be necessary to test this hypothesis.

In summary, vanadyl porphyrin (VO-P) complexes commonly found at trace level in bitumen can be used as intrinsic paramagnetic probes for a non-destructive analysis of the black matter covering ancient Egyptian mummies. Four types of VO-P complexes were identified in HYSCORE spectra by the double-quantum (dq-dq) correlation peaks of ${ }^{14} \mathrm{~N}$ nuclei of porphyrin ligands. These sharp peaks are relatively easy to detect even if the bitumen is mixed with other natural substances (conifer resin, fat, wax etc...). Comparing vanadyl porphyrins of Egyptian black coatings with those of natural bitumen from different geological settings could provide information on the geographical origin of bitumen. As some VO-P complexes may be thermally or chemically unstable, their identification could give information on the thermal/chemical treatments employed in ancient Egypt for the preparation of black coatings of mummies and coffins. ${ }^{1} \mathrm{H}$-hyperfine interaction of VO-P gives information on bitumen content relative to other natural bioorganic substances in the black matters. More important, it indicates also that the mean sizes of bitumen aggregates in these complex mixtures are of the order of a few nanometers. This work and ref. 28 show that combining cw-EPR (in-phase and out-of-phase) with hyperfine spectroscopy (ENDOR, HYSCORE) is a promising tool for a non-destructive exploration of the nanostructure and composition of black coatings of ancient Egyptian mummies and funerary artifacts. The same strategy could be applied to other historical collections such as Islamic metal works for which this black matter could have been widely used as adhesive for metal carving. ${ }^{47}$ EPR tools could also permit to better apprehend local economies, workshop practices and recipes, supply areas as well as trade routes of bituminous materials in the past.

\section{Methods}

All samples (10-20 mg) were inserted into quartz Suprasil EPR tubes. They are shown in Fig.S1. Continuous wave Electron Paramagnetic Resonance (cw-EPR) measurements were performed at room temperature and at $100 \mathrm{~K}$ with a Bruker Elexsys E500 EPR-ENDOR spectrometer operating at about 9.6 GHz (X-band) and $34 \mathrm{GHz}$ (Q-band), equipped with a high sensitivity X-band 4122SHQE/0111 EPR cavity and a Q-band ER5106QTE resonator for both EPR and 
ENDOR. Cw-ENDOR at Q-band was used to measure the hyperfine interaction with ${ }^{1} \mathrm{H}$ nuclei of porphyrin ligands and their molecular environment (see Fig. 2a). The ENDOR spectra were recorded at $100 \mathrm{~K}$ by using a CF935 helium flow cryostat from Oxford Instruments. The radiowaves were amplified by an ENI3100L amplifier, and the ENDOR signals were detected by a $25 \mathrm{kHz}$ frequency modulation of the sweeping rf field, with a modulation depth of $100 \mathrm{kHz}$. The rf was swept in the range 45-60 MHz, centered at the proton Larmor frequency.

EPR spectra at X-band were also recorded by 2-pulse echo field sweep experiment, using the standard Hahn echo sequence $\pi / 2-\tau-\pi$ - $\tau$-echo. The resulting echo-detected absorption EPR spectrum (ED-EPR) was pseudo-modulated to give a first derivative ED-EPR spectrum similar to the cw-EPR spectrum.

HYSCORE experiments were performed at $6 \mathrm{~K}$ with the pulse sequence $\pi / 2-\tau-\pi / 2-\mathrm{t} 1-\pi-\mathrm{t} 2-\pi / 2-$ $\tau$-echo, with pulse lengths of $22 \mathrm{~ns}$ and $44 \mathrm{~ns}$ for $\pi / 2$ and $\pi$ pulses, respectively, and the delay $\tau$ $=200 \mathrm{~ns}$ was chosen as an optimum to prevent blind spot effects. ${ }^{20}$ The spectra were recorded with $256 \times 256$ data points for $\mathrm{t} 1$ and $\mathrm{t} 2$ time domains. The unmodulated part of the echo was removed by second-order polynomial subtraction. Final HYSCORE spectra were obtained by 2D-Fourier transformation of the data set, using a Hamming apodization window function.

EPR and HYSCORE spectra were simulated with the EasySpin toolbox for Matlab (version 5.2.28). ${ }^{30}$

\section{References}

1 Maurer, J.; Möhring, T.; Rullkötter, J.; Nissenbaum, A. Plant lipids and fossil hydrocarbons in embalming material of Roman Period mummies from the Dakhleh Oasis, Western Desert, Egypt. J. Arch. Sci. 29, 751-762 (2002).

2 Buckley, S.A.; Clark, K.A.; Evershed, R.P. Complex organic chemical balms of Pharaonic animal mummies. Nature 43, 294298 (2004).

3 Clark, K.A.; Ikram, S.; Evershed, R.P. The significance of petroleum in in ancient Egyptian mummies. Phil. Trans. R. Soc. A, 374, 20160229 (2016).

4 Fulcher, K. ; Serpico, M. ; Taylor, J.H. ; Stacey, R. Molecular analysis of black coatings and anointing fluids from ancient Egyptian coffins, mummy cases and funerary objects. Proc. Natl. Acad. Sci. U.S.A. 118, 1-10 (2021).

5 Lucas, A; Harris, J.R. Ancient Egyptian Materials and Industries, Histories and Mysteries of Man, Ltd London, IVth ed., 523 p. (1989)

6 Buckley, S.A.; Evershed, R.P. Organic chemistry of embalming agents in Pharaonic and Graeco-Roman mummies. Nature 413, 837-841 (2001).

7 Davis, E. Mummy mania. Chemistry World 8, 48-51 (2011).

8 Spielmann, P.E. To what extent did the ancient Egyptian employ bitumen for embalming. J. Egyptian Archeology 18, 177-180 (1933).

9 Rullkötter, J. Nissenbaum, A. Dead Sea asphalt in Egyptian mummies: molecular evidence. Naturewissenschaften 75, 618621 (1988).

10 Connan, J.; Dessort, D. Du bitume de la Mer Morte dans les baumes d'une momie égyptienne : identification par critères moléculaires. C.R. Acad. Sci. Paris 309, 1665-1672 (1989).

11 Colombini, M.P. ; Modugno, F. ; Silvano, F. ; Onor, M. Characterization of the balm of an Egyptian mummy from the seventh century B.C. Studies in Conservation 45, 19-29 (2000).

12 Harrell, J.A.; Lewan, M.D. Sources of mummy bitumen in ancient Egypt. Archaeometry 44, 285-293 (2002).

13 Mûnnemann, K.; Bôni, T.; Colacicco, G.; Blûmich, B. ; Rûhli, F.J. Noninvasive ${ }^{1} \mathrm{H}$ and ${ }^{23}$ Na nuclear resonance imaging of ancient Egyptian human mummified tissue. Magn. Reson. Imaging 25, 1341-1345 (2007).

14 Özen, A.C.; Ludwig, U.; Öhrström, L.M.; Rûhli, F.J. Comparison of Ultrashort Echo Time Sequences for MRI of an Ancient Mummified Human Hand. Magn. Reson. Med. 75, 701-708 (2016). 
15 Karlik, S.J.; Bartha, R.; Kennedy, K.; Chhem R. MRI and Multinuclear MR Spectroscopy of 3,200 Year-Old Egyptian Mummy Brain; AJR 189, 105-110 (2007).

16 Bertrand P. Electron Paramagnetic Resonance Spectroscopy: Fundamentals. Springer, (2020).

17 Premovic, P.I.; Tonsa, I.R.; Pavlovic, M.S.; Lopez, L.; Lo Monaco, S. Classification of the asphalts and their source rocks from the Dead Sea Basin. Fuel 77, 1769-1776 (1998).

18 Rullkötter, J.; Spiro, B.; Nissenbaum, A. Biological marker characteristics of oils and asphalts from carbonate source rocks in a rapidly subsiding graben, Dead Sea, Israel. Geochim. Cosmochim. Acta 49, 1357-1370 (1985).

19 Baker, E.W.; Louda, J.W. In Biological Markers in the Sedimentary Record; John, R.B. Ed.; Elsevier: Amsterdam, Netherlands, pp 125-225 (1986)

20 Ben Tayeb, K. et al. Applications of pulsed electron paramagnetic resonance spectroscopy to the identification of vanadyl complexes in asphaltene molecules. Part 1: Influence of the origin of the feed. Energy Fuels 29, 4608-4615 (2015).

21 Barwise, A.J.G. Role of Nickel and Vanadium in Petroleum Classification. Energy Fuels 4, 647-652 (1990).

22 Breit, G.N.; Wanty, R.B. Vanadium accumulation in carbonaceous rocks: A review of geochemical controls during deposition and diagenesis. Chem. Geol. 91, 83-97 (1991).

23 Lopez, L.; Lo Monaco, S. Vanadium, nickel and sulfur in crude oils and source rocks and their relationship with biomarkers: Implication for the origin of crude oils in Venezuelan basins. Org. Geochem. 104, 53-68 (2017).

24 Uebersfeld, J. ; Etienne, A.; Combrisson, J. Paramagnetic resonance, a new property of coal-like materials. Nature 174, 614 (1954).

25 Skrypczak-Bonduelle, A. et al. EPR of organic radicals in primitive organic matter : A tool for the search of biosignatures of the most ancient traces of life. Appl. Magn. Reson. 33, 371-397 (2008).

26 Bourbin, M. et al. Dating carbonaceous matter in Archean cherts by Electron paramagnetic resonance. Astrobiology 13, 151$162(2013)$

27 Binet, L.; Gourier, D. ; Derenne, S. ; Robert, F. Heterogeneous distribution of paramagnetic radicals in insoluble organic matter from the Orgueil and Murchison meteorites. Geochim. Cosmochim. Acta 66, 4177-4186 (2002).

28 Dutoit, C.E.; Binet, L.; Fujii, H.; Lattuati-Derieux, A.; Gourier, D. Nondestructive Analysis of Mummication Balms in Ancient Egypt Based on EPR of Vanadyl and Organic Radical Markers of Bitumen. Anal. Chem. 92, 15445-15453 (2020).

29 Schweiger, A.; Jeschke, G. Principles of Pulse Electron Paramagnetic Resonance, Oxford University Press, Oxford, (2001).

30 Stoll, S.; Schweiger, A. EasySpin, a comprehensive software package for spectral simulation and analysis in EPR. J. Magn. Reson. 178, 42-55 (2006).

31 Saraceno, A.J.; Coggeshall, N.D.; Fanale, D.T. An electron paramagnetic resonance investigation of vanadium in petroleum oils. Anal. Chem. 33, 500-505 (1961).

32 Aizenshtat, Z.; Sundararaman, P. Maturation trend in oils and asphalts of the Jordan Rift - Utilization of detailed vanadylporphyrin analysis. Geochim. Cosmochim. Acta. 53, 3185-3188 (1989).

33 Biktagirov, T. et al. In situ identification of various structural features of vanadyl porphyrins in crude oil by high-field (3.4 T) Electron-Nuclear Double Resonance spectroscopy combined with Density Functional Theory calculations. Energy Fuels 31, 1243-1249 (2017).

34 Mannikko, D.; Stoll, S. Vanadyl porphyrin speciation based on submegahertz ligand proton hyperfine couplings. Energy Fuels 33, 4237-4243 (2019).

35 Gourier, D.et al. EPR, ENDOR and HYSCORE study of the structure and the stability of vanadyl-porphyrin complexes encapsulated in silica: Potential paramagnetic biomarkers for the origin of life. J. Phys. Chem. 114, 3714-3725 (2010).

36 Dechaine, G.P.; Gray, M.R. Chemistry and association of vanadium compounds in heavy oil and bitumen, and implications for their selective removal. Energy Fuels 24, 2795-2808 (2010).

37 Kevan, L.; Kispert, L.D. In Electron Spin Double Resonance Spectroscopy; John Wiley \& Sons, New York, 239-251 (1976).

38 Stoll, S.; Calle, C.; Mitrikas, G.; Schweiger, A. Peak suppression in ESEEM spectra of multinuclear spin systems. J. Magn. Reson. 177, 93-101 (2005).

39 Mamin, G.V. et al. Towards the asphaltene structure by electron paramagnetic relaxation studies at high fields (3.4 T). Energy Fuels 30, 6942-6946 (2016).

40 Mullins, O.C. The modified Yen model. Energy Fuels 24, 2179-2207 (2010).

41 Dikanov, S.A.; Xun, L.; Karpiel, A.B.; Tyryshkin, A.M.; Bowman, M.K. Orientationally-selected two-dimensional ESEEM spectroscopy of the Rieske-type iron-sulfur cluster in 2,4,5-Trochlorophenoxyacetate monooxygenase from Burkholderia cepaia AC1100. J. Am. Chem. Soc. 118, 8408-8416 (1996).

42 Reijerse, E.J.; Tyryshkin, A.M.; Dikanov, S.A. Complete determination of nitrogen quadrupole and hyperfine tensors in an oxovanadium complex by simultaneous fitting of multifrequency ESEEM powder spectra. J. Magn. Reson. 131, 295-309 (1998).

43 Garcia-Rubio, I.; Martinez, J.I.; Picorel, R.; Yruela, I. ; Alonso, P.J. HYSCORE spectroscopy in the Cytochrome $b_{559}$ of the photosystem II reaction center. J. Am. Chem. Soc. 125, 15846-15854 (2003).

44 Dikanov, S.A.; Shubin, A.A.; Kounosu, A.; Iwasaki, T.; Samoilova, R.I. A comparative, two-dimensional ${ }^{14}$ N ESEEM characterization of reduced [2Fe-2S] clusters in hyperthermophilic archaeal high- and low-potential Rieske-type proteins. $J$. Biol. Inorg. Chem. 9, 753-767 (2004).

45 Gracheva, I.N. et al. ENDOR study of nitrogen hyperfine and quadrupolar tensors in vanadyl porphyrins of heavy crude oil. Magn. Reson. in Solids 18, 16102 (2016).

46 Fukui K.; Ohya-Nishiguchi H.; Kamada H. Electron Spin Echo Envelope Modulation Study on Oxovanadium(IV)-Porphyrin Complexes: Reinvestigation of Hyperfine and Quadrupole Couplings of Pyrrole Nitrogen. J.Phys.Chem. 97, 11858-11860 (1993).

47 Porter V.; Rosser-Owen M. Metalwork and material culture in the Islamic world art, craft and text. I.B.Tauris Eds. 544 pp (2012). 


\section{Acknowledgment}

CED, DG and LB received funding from Agence Nationale de la Recherche (ANR) under contract $\mathrm{N}^{\circ}$ ANR-17-CE29-000201. The authors are very grateful to the Musée Dobrée in Nantes, the Musée d'Art et d'Histoire in Narbonne, the Musée des Confluences in Lyon and the Chateau-Musée de Boulogne-sur-Mer and more especially to Julie Pellegrin, Camille Broucke, Flore Collette, Elikya Kandot and Gaëlle Etesse who are in charge of collections. In addition, we would like to express our gratitude to Nathalie Balcar, conservation scientist at the C2RMF for the mummy samples. Authors thank CNRS's research federation RENARD (FR3443) for EPR facilities.

\section{Author information}

\section{Corresponding Author}

* E-mail: didier.gourier@ chimieparistech.psl.eu

ORCID Didier Gourier : 0000-0002-9996-9297

\section{Author Contributions}

CED, LB and OA recorded EPR and ENDOR spectra; CED and HV performed pulse-EPR measurements; DG interpreted the results. ALD carried out additional analyses by GC-MS; the manuscript was written by DG with contributions CED, LB and HV. All authors have given approval to the final version of the manuscript.

\section{Competing interests}

The authors declare no competing financial interest.

\section{Supplementary information}

Samples; EPR spectra; ENDOR spectra; Derivation of Equation 1; HYSCORE spectra; Estimation of $2^{\text {nd }}$ order contributions to the ${ }^{14} \mathrm{~N}$ parameters from dq-dq and sq-dq correlation peaks. 


\section{Supplementary Files}

This is a list of supplementary files associated with this preprint. Click to download.

- Supplementarylnformation.pdf

- Figures1.tif

- Figures2.tif

- FigureS3.tif

- Figures4.tif

- Figures5.tif

- Figures6.tif

- FigureS7.tif

- Figures8.tif

- FigureS9.tif 